\title{
Genome scale metabolic modeling reveals the metabolic potential of three Type II methanotrophs of the genus Methylocystis.
}

Sergio Bordel* ${ }^{1,2}$, Yadira Rodríguez ${ }^{1,2}$, Anna Hakobyan $^{3}$, Elisa Rodríguez ${ }^{1,2}$, Raquel Lebrero $^{1,2}$, Raúl Muñoz ${ }^{1,2}$.

1. Departamento de Ingeniería Química y Tecnología del medio ambiente, Escuela de Ingenierías Industriales, Universidad de Valladolid, Spain.

2. Institute of Sustainable Processes, Universidad de Valladolid, Spain.

3. Max Planck Institute for Terrestrial Microbiology, Research group Methanotrophic bacteria and Environmental Genomics/Transcriptomics. Marburg, Germany.

*Author for correspondence: sergio.bordel@uva.es

Keywords: Methanotrophs, metabolism, genome-scale models, Methylocystis. 


\begin{abstract}
Genome Scale Metabolic Models (GSMMs) of the recently sequenced Methylocystis hirsuta and two other methanotrophs from the genus Methylocystis have been reconstructed. These organisms are Type II methanotrophs with the ability of accumulating Polyhydroxyalkanoates under nutrient limiting conditions. For the first time, GSMMs have been reconstructed for Type II methanotrophs. These models, combined with experimental biomass and PHB yields of Methylocystis hirsuta, allowed elucidating the methane oxidation mechanism by the enzyme pMMO (particulate methane monooxygenase) in these organisms. In contrast to Type I methanotrophs, which use the "direct coupling mechanism", Type II methanotrophs appear to use the so called "redox arm mechanism". The utilization of the "redox arm mechanism", which involves the coupling between methane oxidation and complex I of the respiratory chain, was confirmed by inhibition of complex I with catechol. Utilization of the "redox arm" mechanism leads to lower biomass yields on methane compared to Type I methanotrophs. However, the ability of Type II metanotrophs to redirect high metabolic carbon fluxes towards acetoacetylCoA under nitrogen limiting conditions makes these organisms promising platforms for metabolic engineering.
\end{abstract}

\title{
1. Introduction
}

Methanotrophic organisms, able to use methane as a carbon and energy source, are attracting a growing attention as potential cell factories (Strong et. al., 2016). Methane has been recently identified as a cost effective feedstock for microbial fermentations as a result of the low price of natural gas and the residual nature of some $\mathrm{CH}_{4}$-laden emissions (Comer et. al., 2017). Indeed, this gas has the potential of becoming a virtually free substrate based on the fact that methane is originated in most waste treatment processes and more than 70 million tonnes of $\mathrm{CH}_{4}$ are being yearly released into the atmosphere worldwide (Abbasi et. al., 2012). The replacement of glucose by methane would also have positive environmental and societal impacts. Methane is a greenhouse gas and its utilization as a feedstock would constitute a carbon sink. However, to this date, only a few methane-based industrial bioproducts have reached the market, and none of them involves metabolically engineered strains. 
Nevertheless, some interesting works on metabolic engineering of methanotrophs have been already conducted as a result of the development of adapted genetic tools and the reconstruction of Genome Scale Metabolic Models (GSMMs) for these organisms (Kalyuzhnaya et. al., 2015). Most of these examples have been carried out in Type I methanotrophs, which assimilate methane by oxidizing it to formaldehyde and condensing formaldehyde with ribulose monophosphate, resulting in fructose-6-phosphate production. The first published GSMM of a methanotroph was that of Methylomicrobium buriatense (de la Torre et. al., 2015). M. buriatense was also the first methanotroph to be metabolically engineered to produce lactate (Henard et. al., 2016), thanks to the development of techniques for its genetic manipulation (Puri et. al., 2015). A GSMM of Methylomicrobium alcaliphilum has been also developed (Akberdin et. al., 2018) and used to optimize the production of 2,3-butanediol (Nguyen et. al., 2018) by applying the OptGene algorithm (Patil et. al., 2005). The two previously mentioned GSMMs allowed to elucidate the methane oxidation mechanism of the two mentioned Methylomicrobium species, which in both cases appeared to be the so called "direct coupling". The first step in methane degradation is the oxidation of methane to methanol by a membrane bound methane monooxygenase (pMMO), with the consumption of a molecule of oxygen and the oxidation of a reduced electron donor. "Direct coupling" is the mechanism by which the mentioned electron donor is reduced back coupled to the oxidation of methanol to formaldehyde by the enzyme Methanol Dehydrogenase (MeDH). The identification of the electron donor to pMMO has been for a long time an unsolved problem in the study of methanotrophs. A summary of the different theories explaining the mechanisms of methane oxidation can be found in the review by Kalyuzhnaya and coworkers (Kalyuzhnaya et. al., 2015). The identification of the correct methane oxidation mechanism is essential for the design of metabolic engineering strategies, as it has a deep impact on the demand for redox cofactors, which interplay with all the metabolic pathways of the cell.

This study presents, to the best of our knowledge, the three first GSMMs of Type II methanotrophs reported in literature. These organisms use the serine cycle for methane 
assimilation, which results in a relatively high flux through Acetyl-CoA (Kalyuzhnaya et. al., 2015) and might make them particularly suitable for the production of lipid derivatives such as biofuels or carotenoids. Type II methanotrophs from the genera Methylocystis, Methylosinus and Methylocella are also able to synthesize polyhydroxyalkanoates (PHAs) under conditions of nutrient limitation (Pieja et al., 2017). Among them, Methylocystis hirsuta has been found to accumulate polyhydroxybutyrate (PHB) up to $45 \%$ of its total biomass (García-Pérez et. al., 2018). In addition to the commercial interest of PHAs as biodegradable plastics, the high flux towards acetoacetyl-CoA of these organisms could potentially be deviated towards butanol or mevalonate derivatives (valencene, geraniol etc.), which makes them promising future cell factories. Type II methanotrophs exhibit lower methane yields than those of Type I methanotrophs, this phenomenon has been attributed to a "relatively expensive route for $\mathrm{C} 1$ assimilation", the serine pathway (Kalyuzhnaya et. al., 2015). However, this statement cannot be fully proven without using GSMMs. In fact, the GSMMs reconstructed in this work, revealed that the most likely cause for this lower yield is a different mechanism of methane oxidation (the so called "redox arm" instead of "direct coupling").

This study aimed at constructing GSMMs of the recently sequenced Methylocystis hirsuta CSC1 (Bordel et. al., 2018), as well as of its close relative species Methylocystis sp. SC2 and Methylocystis sp. SB2. In this context, $M$. sp. SC2 was the first Type II methanotroph genetically engineered (Baani and Liesack, 2008) and has shown a versatile metabolism, including a complete denitrification pathway (Dam et. al., 2013), which is truncated in $M$. hirsuta (Bordel et. al., 2018). On the other hand, $M$. sp. SB2 is a facultative methanotroph able to grow in 2-carbon substrates such as acetate and ethanol (Im et. al., 2011). In this work, the GSMM of Methylocystis hirsuta has been validated using experimental measurements of growth yields, methane and oxygen consumption, and PHB production. The similarities and differences of the metabolic network of Methylocystis hirsuta with its two close relatives are also discussed.

\section{Materials and methods}




\subsection{Reconstruction of GSMMs}

The genome of Methylocystis hirsuta CSC1 was previously sequenced, assembled, and annotated (Bordel et. al., 2018), and has been deposited in GenBank with the accession number QWDD00000000 (BioProject: PRNJ487728). The genome of Methylocystis sp. SC2 (Dam et. al., 2012) was obtained from GenBank with accession number NC_018485 (BioProject: PRJNA224116), while that of Methylocystis sp. SB2 was also obtained from GenBank, with accession number GCA_000499825 (BioProject: PRJNA219752). Draft models were generated using the SEED server (Overbeek et. al., 2005) based on a previous annotation with RAST (Overbeek et. al., 2014). Each draft was manually curated by checking all the gene-reaction associations and correcting dubious annotations. For example, the SEED reaction with identifier rxn00470_c0, that corresponds to ornithine decarboxylation, was associated to the gene with identifier 369798.4.peg.465, which was annotated by RAST as diaminopimelate decarboxylase (EC 4.1.1.20), the corresponding EC entry was checked in KEGG and it was found not to be associated to ornithine decarboxylation, therefore the annotation of this reaction was changed manually to the gene 369798.4.peg.3994, annotated by RAST as ornithine decarboxylase (EC 4.1.1.17). Another example of annotation corrected manually, was nitrate reductase, with SEED identifier rxn10121_c0, which was associated to the gene 369798.4.peg.730, annotated by RAST as a nitrite reductase subunit (EC 1.7.1.4), this association was changed manually to the genes 369798.4.peg.732 and 369798.4.peg.733, which were annotated by RAST as nitrate reductases (EC 1.7.99.4). The models were manipulated in SBML format using the Python library COBRApy (Ebrahim et. al., 2013). Reaction directionality was modified in order to avoid unfeasible ATP production, so that the model only simulates ATP production in the respiratory chain and in the lower glycolysis. Directionality of the reactions involving proton transport was also modified in order to guarantee that no proton flux towards the exterior of the cell is allowed if it is not mediated by the components of the respiratory chain or associated to some energy cost. This was done in order to avoid proton flux against its concentration gradient. The biomass equations used were those generated by SEED. 


\subsection{Strain, chemicals and culture conditions.}

The strain Methylocystis hirsuta CSC1 was obtained from the DSMZ culture collection (DSM18500). The strain was cultured in Whittenbury nitrate mineral medium (pH 6.8) (Whittenbury et. al., 1970) or alternatively Whittenbury ammonium mineral medium or nitrogen free Whittenbury mineral medium for the corresponding experiments. M. hirsuta $\mathrm{CSC} 1$ growth and PHB accumulation experiments were carried out in $2.15 \mathrm{~L}$ serum bottles closed with butylrubber stoppers and aluminum crimp seals (incubation at $25^{\circ} \mathrm{C}$ and $300 \mathrm{rpm}$ ). Each flask contained $400 \mathrm{~mL}$ of mineral medium and a headspace containing mixtures of synthetic biogas (70\% $\mathrm{CH}_{4}, 30 \% \mathrm{CO}_{2}$ ) and $\mathrm{O}_{2}$ from Abelló Linde S.A. (Barcelona, Spain). Catechol was purchased from Sigma Aldrich®, St. Louis, USA. Headspace mixtures with 2:1, 1.5:1 and 1:1 $\mathrm{O}_{2}: \mathrm{CH}_{4}$ molar ratios were prepared in $25 \mathrm{~L}$ Tedlar bags (Sigma Aldrich ${ }^{\circledR}$, St. Louis, USA) and subsequently pumped into the bottles to replace the original air headspace. The time course of the headspace composition and biomass concentration were monitored by GC-TCD and optical density (after performing a calibration curve) respectively. The growth rates were obtained from the slopes of the logarithms of the biomass concentration with respect to time, while biomass yields and oxygen-methane consumption ratios were obtained from the slope of the biomass with respect to the consumed methane and from the slope of the consumed oxygen with respect to the consumed methane. Methane specific consumption rate was obtained by dividing the specific growth rate by the yield of biomass on methane. Total amounts of oxygen and methane were calculated from their gas concentrations taking into account the gas and liquid volumes in the flasks and the solubility of each gas at $25^{\circ} \mathrm{C}$. Total $\mathrm{CO}_{2}$ accumulation was calculated based on the $\mathrm{pH}$, the $\mathrm{CO}_{2}$ concentration in the headspace, the Henry's law constant and the equilibrium constant with $\mathrm{HCO}_{3}{ }^{-}$and $\mathrm{CO}_{3}{ }^{2-}$.

Experiments assessing $M$. hirsuta growth on acetate and the inhibition of its respiratory chain by catechol were carried out at $25^{\circ} \mathrm{C}$ in $125 \mathrm{~mL}$ serum bottles crimp sealed under sterile conditions with $50 \mathrm{~mL}$ of NMS and $20 \mathrm{~mL}$ NMS, respectively, under a $\mathrm{O}_{2}: \mathrm{CH}_{4}$ atmosphere $(2: 1$ molar ratio). 
Methylomicrobium alcaliphilum 20Z, a type I methanotroph, was purchased from DSMZ (DSM-19304). M. alcaliphilum was grown on a high alkalinity (pH 9) as described previously (Kalyuzhnaya et. al. 2008). The catechol inhibition tests were carried out under the same conditions as for $M$. hirsuta.

Control experiments with Methylocystis sp. SC2 were carried out at Prof. Liesack's laboratory (Max Planck institute for terrestrial microbiology) under culture conditions previously reported for the mentioned microorganism (Dam et. al., 2012)

\subsection{Analytical methods.}

The headspace gas concentrations $\left(\mathrm{CH}_{4}, \mathrm{O}_{2}\right.$ and $\left.\mathrm{CO}_{2}\right)$ were measured by gas chromatography in a Bruker 430 GC-TCD (Bruker, Palo Alto, USA) with two columns: a CP-Molsieve 5A (15 m × $0.53 \mathrm{~mm} \times 10 \mathrm{~mm})$ and a CP-PoraBOND Q $(25 \mathrm{~m} \times 0.53 \mathrm{~mm} \times 10 \mathrm{~mm})$. Biomass concentration was estimated using $\mathrm{OD}_{600}$, which was previously correlated with total suspended solids (TSS) as described previously (López et. al., 2014). Nitrate concentrations were measured by HPLC-IC using a Waters 432 conductivity detector (Waters, USA). The specific nitrate production rate for the experiment in which ammonium was used as nitrogen source was calculated multiplying the slope of nitrate concentration versus biomass by the specific growth rate.

The PHB content was extracted from the biomass as described by Zuniga and coworkers (Zuniga et. al., 2011). After extraction and hydrolysis, hydroxybutyrate was measured using an Agilent 7820A GC coupled with a 5977E MSD (Agilent technologies, Santa Clara, USA) equipped with a DB-wax column $(30 \mathrm{~m} \times 250 \mu \mathrm{m} \times 0.25 \mu \mathrm{m})$. The detector and injector temperatures were kept constant at $250^{\circ} \mathrm{C}$ and the oven temperature was increased from $40{ }^{\circ} \mathrm{C}$ to $200{ }^{\circ} \mathrm{C}$ at $10{ }^{\circ} \mathrm{C}$ per minute and maintained at $200{ }^{\circ} \mathrm{C}$ for 2 minutes, before being increased again at $5{ }^{\circ} \mathrm{C}$ per minute until reaching $240{ }^{\circ} \mathrm{C}$. Acetaldehyde was measured using an Agilent $1200 \mathrm{HPLC}$ with $355 \mathrm{~nm}$ UV detector and a C18 column $(250 \times 4.6 \mathrm{~mm})$ at $30^{\circ} \mathrm{C}$. 


\section{Results}

\subsection{Genome Scale Metabolic Models}

After the manual curation of the SEED models, three GSMMs whose statistics are represented in Table 1 were obtained. Essential reactions are defined as those whose absence makes the model unable to produce biomass using methane as a carbon and energy source.

Table 1. Sizes of the reconstructed GSMMs

\begin{tabular}{lcccc}
\hline & Genes & Reactions & Metabolites & Essential reactions \\
\hline M. hirsuta & 2748 & 1399 & 1460 & 383 \\
M. sp. SC2 & 2251 & 1449 & 1434 & 381 \\
M. sp. SB2 & 2281 & 1380 & 1453 & 374 \\
\hline
\end{tabular}

An ATP maintenance reaction was added to all the models, as well as two alternative methane monooxygenase reactions with identifiers pMMO1 and pMMO2, respectively. Reaction pMMO1 uses cytochrome-c as co-factor and is aimed to represent the direct coupling mechanism for methane oxidation, while pMMO2 uses ubiquinone as redox cofactor and is aimed to represent the redox-arm mechanism. Serine-glyoxylate aminotransferase (with EC 2.6.1.45), which is present in the three genomes and belongs to the serine cycle, was added manually. A PHB synthesis reaction, representing the polymerization of (S)-3-hydroxybutyrilCoA to form PHB, was also added in order to account for the PHB accumulation observed in the members of the genus Methylocystis, even if no clear annotation was found in the genome. The reaction with identifier rxn10113_c0, representing complex III in the respiratory chain was modified to represent an electron transfer between ubiquinol and cytochrome-c (in the draft model this reaction was written lumped to the final electron transfer to $\mathrm{O}_{2}$ ). If this reaction is allowed to proceed backwards, this mimics the so called uphill electron transfer hypothesis (Kalyuzhnaya et. al., 2015). The reconstructed models in SBML format and in tab separated format, as well as the connectivity information for the most connected metabolites and the lists of essential reactions, have been made publicly available at 
https://github.com/SergioBordel/ModelsMethanotrophs. The models in SBML format have also been deposited in BioModels (Chelliah et. al., 2015) with identification numbers: MODEL1812120001, MODEL1812120002 and MODEL1812120003.

Figure 1 represents the overlap between the metabolic networks of the three species herein considered. The three species shared more than $90 \%$ of their metabolic reactions and metabolites, and the theoretical biomass and PHB yields on methane were identical.

A Shared metabolic reactions

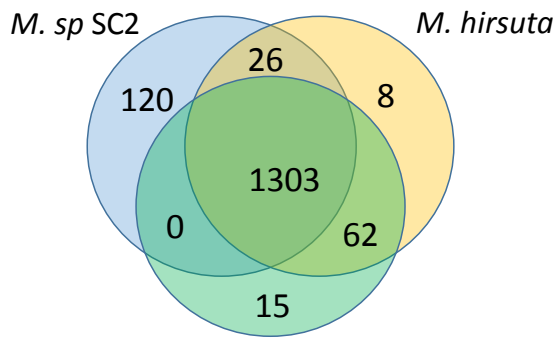

M. $s p$ SB2

C

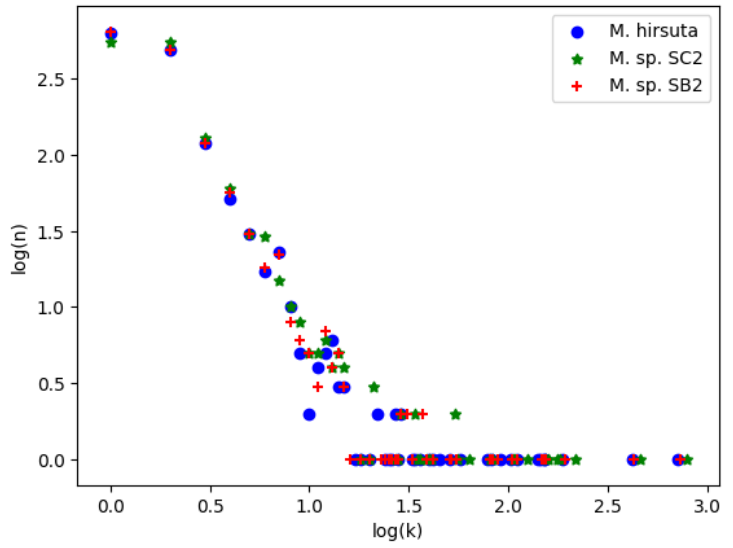

B Shared metabolites

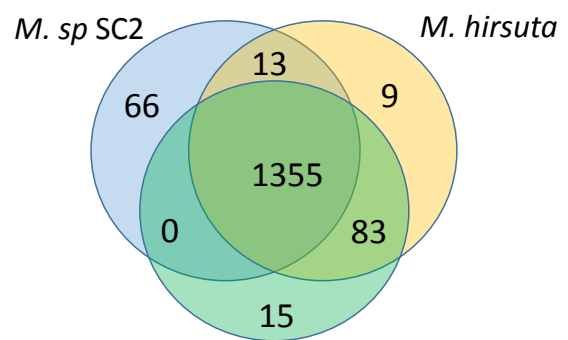

M. $s p \mathrm{SB} 2$

D

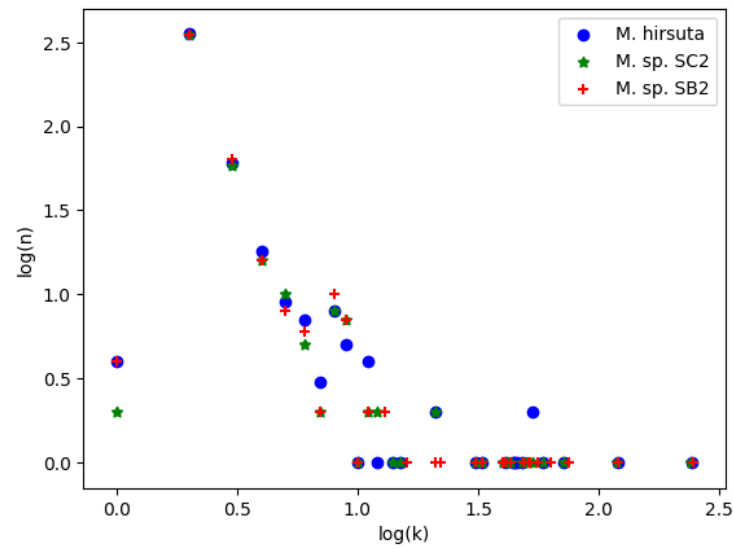

Figure 1. Topological characteristics of the reconstructed metabolic networks. The Venn diagrams show the overlap in reactions (A) and metabolites (B) among the three Methylocystis GSMMs. The scatter plots show in logarithmic scales the number ( $\mathrm{n}$ ) of metabolites with connectivity $\mathrm{k}$ (participating in $\mathrm{k}$ metabolic reactions), for the whole network (C) and for the sub-network of flux-carrying reactions during biomass optimization (D). The plots show a scale free topological structure, with a parameter $\gamma$ equal to 2.5 . Highly connected metabolites, such as energy and redox cofactors, constitute outliers.

Sorbitol was identified among the metabolites with potential commercial interest specific to a single species. This metabolite was found only in Methylocystis hirsuta due to the presence of a sorbitol dehydrogenase (EC 1.1.1.14), which was absent in the two other species and could 
catalyze the transformation of D-fructose into sorbitol with the consumption of a NADH molecule. Other metabolites specific to $M$. hirsuta were cyromazine (an insecticide) and its synthesis intermediates such as cyanuric acid. However, cyromazine synthesis was annotated as being the result of the promiscuous transaminase activity of a single dCMP deaminase (EC 3.5.4.45), which makes the presence of this pathway uncertain.

Methylocystis sp. SB2 contained the chelating agent Mg protoporphyrin IX, and its synthesis intermediates deoxylimonate, chlorophyllide, and protochlorophyllide, within its specific metabolites. In this case there were 12 specific genes responsible for this pathway, which were only present in $M$. sp. SB2 and included: Mg protoporphyrin IX monomethyl ester oxidative cyclase (EC 1.14.13.81), Mg protoporphyrin IX O-methyltransferase (EC 2.1.1.11), protoporphyrin IX Mg-chelatase subunits H, I and D (EC 6.6.1.1), lysophospholipase (EC 3.1.1.5), etc. The presence of these genes suggested a higher ability of Methylocystis $s p$. SB2 to uptake $\mathrm{Mg}$ from the extracellular medium.

The species Methylocystis sp. SC2 exhibited 66 specific metabolites and 120 specific reactions, including the enzymes acyl-phosphate glycerol-3-phosphate O-acyltransferase PlsY or methylmalonyl-CoA mutase (EC 5.4.99.2). An interesting specific metabolite found only in $M$. sp. SC2, together with its biosynthesis intermediates, was cobalt-precorrin, which could indicate a higher capability of Co assimilation compared to the other two species. Aniline, which is the product of an aromatic-L-amino-acid decarboxylase (EC 4.1.1.28), was identified among the metabolites with potential industrial interest found in Methylocystis sp. SC2 genome.

From a topological point of view all the networks showed a scale free structure in which the number of metabolites (n) participating in $\mathrm{k}$ reactions was proportional to $\mathrm{k}^{-\gamma}$, with the parameter $\gamma$ equal to 2.5. The most highly connected metabolites such as water, protons, phosphate and energy or redox cofactors, were outliers with higher abundance than what could be expected from a scale free statistical distribution. This is a universal feature of metabolic networks, which has been observed in organisms as different as E. coli, S. cerevisiae, H. pilori, S. aureus, etc. (Becker, et. al., 2006). The same pattern was observed if only the metabolic subnetwork, whose 
reactions carry a non-zero flux under optimal growth conditions, were analyzed (only the reactions that carry flux when the biomass production was optimized, and their metabolites were considered). In this case, the metabolites participating in only one reaction were underrepresented, as those are the metabolites secreted or up-taken from the medium.

\subsection{Model validation.}

The theoretical yields on methane predicted by the metabolic model were compared to experimental biomass and PHB production yields of M. hirsuta.

\subsubsection{Growth yields and oxygen utilization.}

Different methane oxidation mechanisms result in different growth yields and oxygen demands. For example, the Type I methanotroph Methylomicrobium alcaliphilum was recently found to rely on "direct coupling" by comparing its experimental oxygen-methane consumption ratio with the simulations of different mechanisms using a GSMM (Akberdin et. al., 2018).

M. hirsuta growth experiments were carried out with headspace $\mathrm{O}_{2}: \mathrm{CH}_{4}$ molar ratios of $2: 1$, 1.5:1 and $1: 1$ in order to test if the headspace composition had an impact on the oxygenmethane consumption ratios. Nitrate was used as a nitrogen source (since ammonium results in competitive inhibition of methane monooxygenase as discussed later on). The observed growth rates, yields and oxygen-methane consumption rations are reported in Table 2 (error intervals represent standard deviations among 3 biological replicates).

Table 2. Experimental growth rates, yields and oxygen-methane consumption ratios

\begin{tabular}{cccc}
\hline $\mathbf{O}_{2}: \mathbf{C H}_{4}$ & $\begin{array}{c}\boldsymbol{\mu} \\
\left(\mathbf{d a y}^{-\mathbf{1}}\right)\end{array}$ & $\begin{array}{c}\mathbf{Y} \\
\left(\mathrm{g}-\mathrm{DW} / \mathbf{m o l C H}_{4}\right)\end{array}$ & $\begin{array}{c}\text { Consumption ratio } \\
\left(\mathbf{m o l O}_{\mathbf{2}} / \mathbf{m o l C H}_{4}\right)\end{array}$ \\
\hline $\mathbf{2 : 1}$ & $1.2 \pm 0.01$ & $7.99 \pm 0.005$ & $1.46 \pm 0.05$ \\
$\mathbf{1 . 5 : 1}$ & $1.11 \pm 0.01$ & $7.47 \pm 0.085$ & $1.44 \pm 0.06$ \\
$\mathbf{1 : 1}$ & $1.11 \pm 0.02$ & $7.62 \pm 0.145$ & $1.43 \pm 0.02$ \\
\hline
\end{tabular}


No significant differences among the three experiments were obtained (ANOVA test). In particular, oxygen and methane were consumed with the same stoichiometric ratio until the limiting substrate in the headspace was fully depleted.

In order to model the theoretical yields and consumption ratios in M. hirsuta, the maintenance ATP consumption rate was initially estimated by following the oxygen consumption rate of a culture in the absence of carbon source. The observed specific oxygen consumption rate was $6 \pm 1 \mathrm{mmolO}_{2} \mathrm{~g}-\mathrm{DW}^{-1} \mathrm{day}^{-1}$. Assuming a typical P/O ratio of 2.5 , the maintenance ATP consumption rate was estimated at $30 \mathrm{mmol}$ ATP $\mathrm{g}-\mathrm{DW}^{-1} \mathrm{day}^{-1}$. This value was imposed as a constraint in the model. Growth rates were predicted using the reconstructed model for $M$. hirsuta by setting the specific methane uptake rate to its average experimental value of 150 $\mathrm{mmolCH}_{4} \mathrm{~g}-\mathrm{DW}^{-1}$ day $^{-1}$. Simulations were carried out under the "direct coupling" assumption (allowing flux in the pMMO1 reaction), the "uphill electron transfer" assumption (allowing the reaction catalyzed by complex III of the respiratory chain to proceed in its reverse direction) and finally the "redox arm" assumption (with an irreversible complex III and methane oxidation taking place in the reaction pMMO2). Figure 2 shows the predictions in each scenario compared to the experimental observations. 

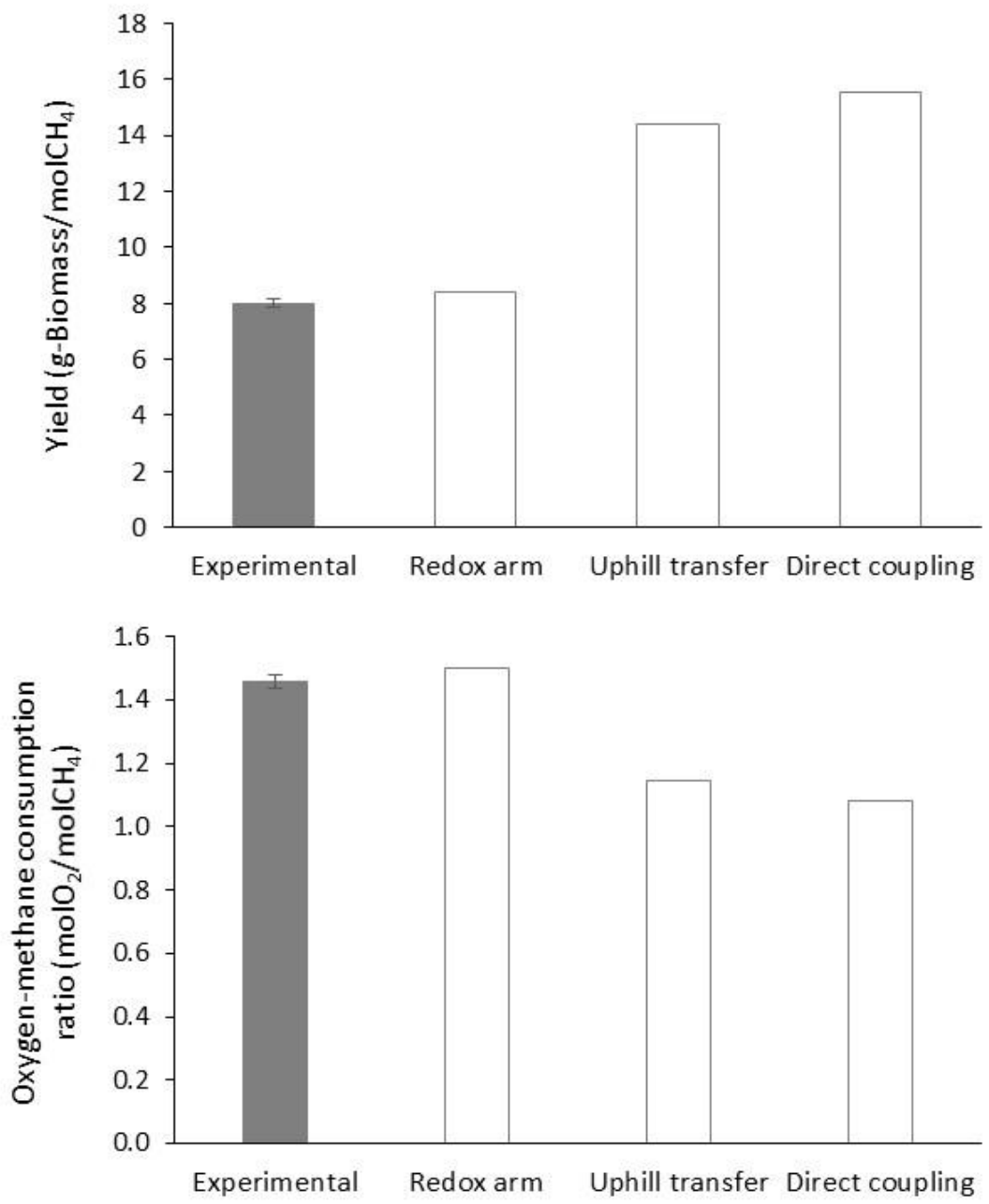

Figure 2. Experimental and theoretical biomass yields (A) and oxygen-methane consumption ratios (B) predicted using the "redox arm", "uphill electron-transfer" and "direct coupling" mechanisms. Grey bars represent experimental measurements with error bars corresponded to the observed standard deviation of the measurements (for 3 biological replicates). White bars correspond to model predictions for each of the three considered methane oxidation mechanisms, using the GSMM of Methylocystis hirsuta.

The experimental results fit remarkably well with the predictions using the "redox arm" mechanism. The oxygen-methane consumption rates predicted by the redox arm mechanism was 1.5. This contrasts with the results obtained with Type I methanotrophs (de la Torre et. al., 2015; Akberdin et. al., 2018), where the experimental results were consistent with the assumption of the "direct coupling" mechanism. Figure 3 illustrates the three potential methane oxidation mechanisms. 

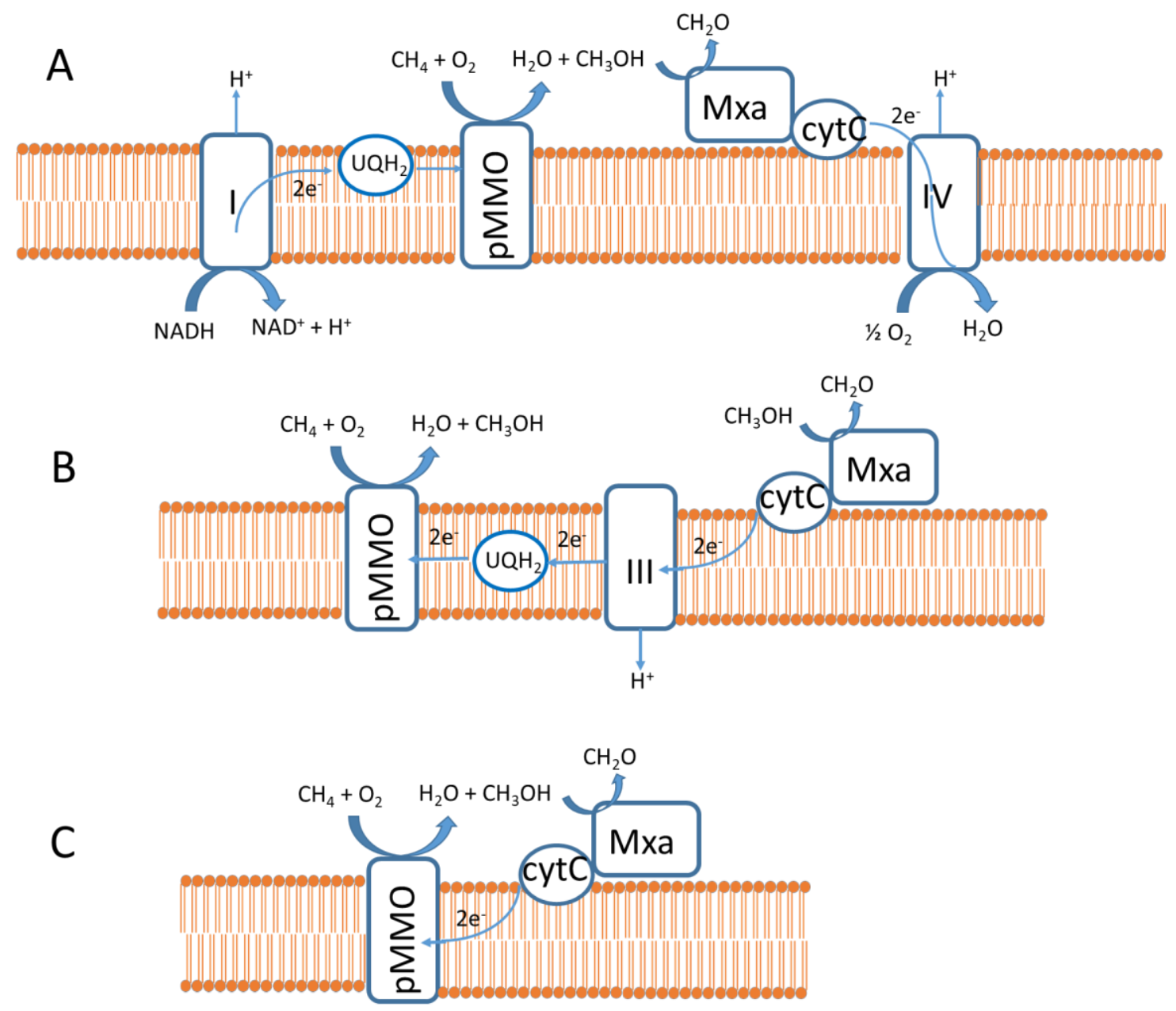

Figure 3. Alternative mechanisms of methane oxidation. (A) "redox arm" mechanism in which ubiquinone acts as electron supplier to pMMO, these electrons originating ultimately from NADH via the complex I of oxidative phosphorylation. (B) "uphill-electron transfer" mechanism where the electrons supplied to pMMO by ubiquinone originate from cytochrome-c via the reverse operation of complex III. (C) "direct coupling" mechanism where cytochrome-c supplies directly electrons to pMMO.

\subsubsection{Complex I inhibition affects methane oxydation.}

Under the redox arm mechanism, methane consumption is coupled to the reduction of ubiquinone by the complex I of the respiratory chain. Therefore, complex I inhibition would result in the inhibition of methane oxidation. Under the direct coupling hypothesis, even in the case of an inactive complex I, methane oxidation to formaldehyde could still be carried out autonomously, without the necessity of NADH inputs. On the other hand, complexes II, III and IV alone, are expected to be able to sustain respiration. Even if the inhibition of complex I will decrease the global energetic efficiency of the oxidative phosphorylation. 
Catechol has been reported to inhibit complex I at $0.4 \mathrm{mM}$ concentrations (Degli Esposti, 1998). In order to validate the existence of a redox arm mechanism in M. hirsuta (and its close relatives, given the fact that they have identical pMMO complexes), catechol was used to inhibit complex I and its effects on methane oxidation were monitored.

The same experiment was performed with Methylomicrobium alcaliphilum, a type I methanotroph for which the direct coupling mechanism has been previously established (Akberdin et. al., 2018). In this case, catechol is not expected to inhibit the oxidation of methane to glyceraldehyde and slowing down the rate of respiration could result in accumulation of acetaldehyde in the growth medium.

Aliquots of $20 \mathrm{~mL}$ of a $M$. hirsuta culture in the exponential growth phase (with a dry weight concentration of $205 \mathrm{mg} \mathrm{L}^{-1}$ ) were placed in $125 \mathrm{~mL}$ serum flasks. A first test series was carried out (in triplicate) by adding catechol to the culture broth at $0.4 \mathrm{mM}$, while a second series (in triplicate) without catechol was used as a control. Methane and oxygen headspace concentrations were monitored over time and their specific biodegradation rates were calculated from their initial slopes. Liquid samples were taken after 24 hours in order to measure possible glyceraldehyde accumulation. The same experimental procedure was performed with $20 \mathrm{~mL}$ aliquots of M. alcaliphilum in the exponential growth phase with dry weight concentrations of $170 \mathrm{mg} \mathrm{L}^{-1}$.

The specific methane elimination rates, oxygen-methane consumption ratios and specific glyceraldehyde accumulation rates are summarized in table 3. The oxygen-methane consumption ratios without catechol inhibition were consistent with the redox-arm (for $M$. hirsuta) and the direct coupling mechanism (for M. alcaliphilum). A specific methane oxidation rate of $4.95 \mathrm{mmol} \mathrm{g-DW}{ }^{-1} \mathrm{day}^{-1}$ was observed for M. alcaliphilum (compared to 181 for $M$. hirsuta). This was also consistent with the low specific growth rate observed during the pregrowth of the inoculum ( 0.1 day $^{-1}$ compared to 1.1 for M. hirsuta). Catechol addition resulted in a methane oxidation rate of $9 \%$ its initial value for the type II methanotroph $M$. hirsuta, while 
the type I methanotroph $M$. alcaliphilum was still able to oxydize methane at $54 \%$ of its initial rate.

The concentration of glyceraldehyde was also measured. Assuming that in the case of a direct coupling mechanism, catechol addition will have a stronger effect on the downstream consumption of glyceraldehyde than on its production, a higher accumulation rate of this compound is expected due catechol addition to M. alcaliphilum. After 24 hours of catechol addition, the concentration of formaldehyde in the culture broth of $M$. alcaliphilum increased from zero (at the beginning of the experiment) to values between 0.36 and $0.65 \mathrm{mg} \mathrm{L}^{-1}$, while no formaldehyde accumulation was observed for the control. In the case of M. hirsuta, glyceraldehyde was already present at the beginning of the experiment at $2 \mathrm{mg} \mathrm{L}^{-1}$ and in some of the biological replicates it decreased while in others increased. No statistically significant difference was observed due to catechol addition (average specific accumulation rates and standard deviations are shown in table 3).

Both organisms showed higher oxygen-methane consumption rates under catechol inhibition. This is likely due to lower efficiencies in proton extrusion caused by complex I inhibition and to proton leaks caused by membrane disruption. In any case, the oxygen-methane consumption ratio of M. alcaliphilum inhibited with catechol is still lower than the value for the non-inhibited M. hirsuta. All these results come to support the hypothesis of $M$. hirsuta relying on a redoxbranch mechanism for methane oxidation, in contrast to the type I methanotroph $M$. alcalyphilum, which uses a direct coupling mechanism.

Table 3: Effects of catechol at $0.4 \mathrm{mM}$ on the specific $\mathrm{CH}_{4}$ consumption rates, $\mathrm{O}_{2}: \mathrm{CH}_{4}$ consumption ratios and formaldehyde accumulation rates of Methylocystis hirsuta and Methkylomicrobium alcaliphilum. Error intervals are standard deviations (3 biological replicates).

\begin{tabular}{ccccc}
\hline & $\begin{array}{c}\text { M. } \\
\text { hirsuta } \\
(\text { control })\end{array}$ & $\begin{array}{c}\text { M. hirsuta } \\
(\text { catechol 0.4 } \\
\mathbf{m M})\end{array}$ & $\begin{array}{c}\text { M. } \\
\text { alcaliphilum } \\
\text { (control) }\end{array}$ & $\begin{array}{c}\text { alcaliphilum } \\
\text { (catechol 0.4 } \\
\text { mM) }\end{array}$ \\
\hline $\begin{array}{c}\text { Specific } \mathrm{CH}_{4} \text { consumption rate } \\
\left(\mathrm{mmol} \mathrm{g-DW}^{-1} \text { day }^{-1}\right)\end{array}$ & $181 \pm 1$ & $17 \pm 5$ & $4.95 \pm 0.18$ & $2.65 \pm 0.4$
\end{tabular}


Specific $\mathrm{CH}_{2} \mathrm{O}$ accumulation rate $\left(\mathrm{mmol} \mathrm{g-DW}{ }^{-1}\right.$ day $\left.^{-1}\right)$

$0.12 \pm 0.31 \quad 0.02 \pm 0.35$

$0.12 \pm 0.05$

$\mathrm{O}_{2}: \mathrm{CH}_{4}$ consumption ratio

$1.58 \pm 0.02 \quad 1.8 \pm 0.1$

$1.16 \pm 0.01$

$1.37 \pm 0.02$

\subsubsection{Influence of the nitrogen source.}

The experimental and predicted biomass yields of $M$. hirsuta when ammonium was used as nitrogen source instead of nitrate were herein compared. Nitrogen is typically incorporated into amino acids in the same oxidation state than ammonium, and therefore, no redox equivalents for its reduction are necessary, as it is the case for nitrate, which needs to be reduced to ammonium before being incorporated into amino acids, thus higher biomass yields are expected using ammonium as nitrogen source. However, ammonium is known to inhibit pMMO via competitive inhibition (Campbell et. al., 2011), as pMMO can also catalyze the oxidation of ammonium to hydroxylamine. Indeed, when M. hirsuta was grown in an ammonium mineral medium with a molar $\mathrm{O}_{2}: \mathrm{CH}_{4}$ ratio of $1: 1$ in the headspace, the experimentally observed growth rate was $0.76 \pm 0.02$ day $^{-1}$ compared to $1.11 \pm 0.02$ day $^{-1}$ when nitrate was used as nitrogen source. Interestingly, nitrate was accumulated in the cultivation medium at a rate of $1.5 \pm 0.1 \mathrm{mmol} \mathrm{g}$ $\mathrm{DW}^{-1}$ day $^{-1}$ during the experiment carried out with ammonium as nitrogen source, which suggested that the hydroxylamine produced by the pMMO was further oxidized to nitrite and finally to nitrate. This nitrate accumulation rate revealed that the rate of ammonium oxidation by pMMO was only $2.3 \%$ of the methane oxidation rate (it was assumed that all the accumulated nitrate is being originated from $\mathrm{NH}_{4}{ }^{+}$oxidized by pMMO to hydroxylamine and subsequently to nitrite and nitrate). However, the competitive inhibition mediated by the presence of ammonium caused the specific methane oxidation rate to drop from $137 \pm 0.7 \mathrm{mmol}$

$\mathrm{g}-\mathrm{DW}^{-1}$ day $^{-1}$ to only $67 \pm 5 \mathrm{mmol} \mathrm{g}-\mathrm{DW}^{-1}$ day $^{-1}$. Finally, when setting the experimental methane uptake rate and the nitrate production rate to their experimental values, the predicted and experimental biomass productions in M. hirsuta cultures showed a good agreement (Figure 4). 


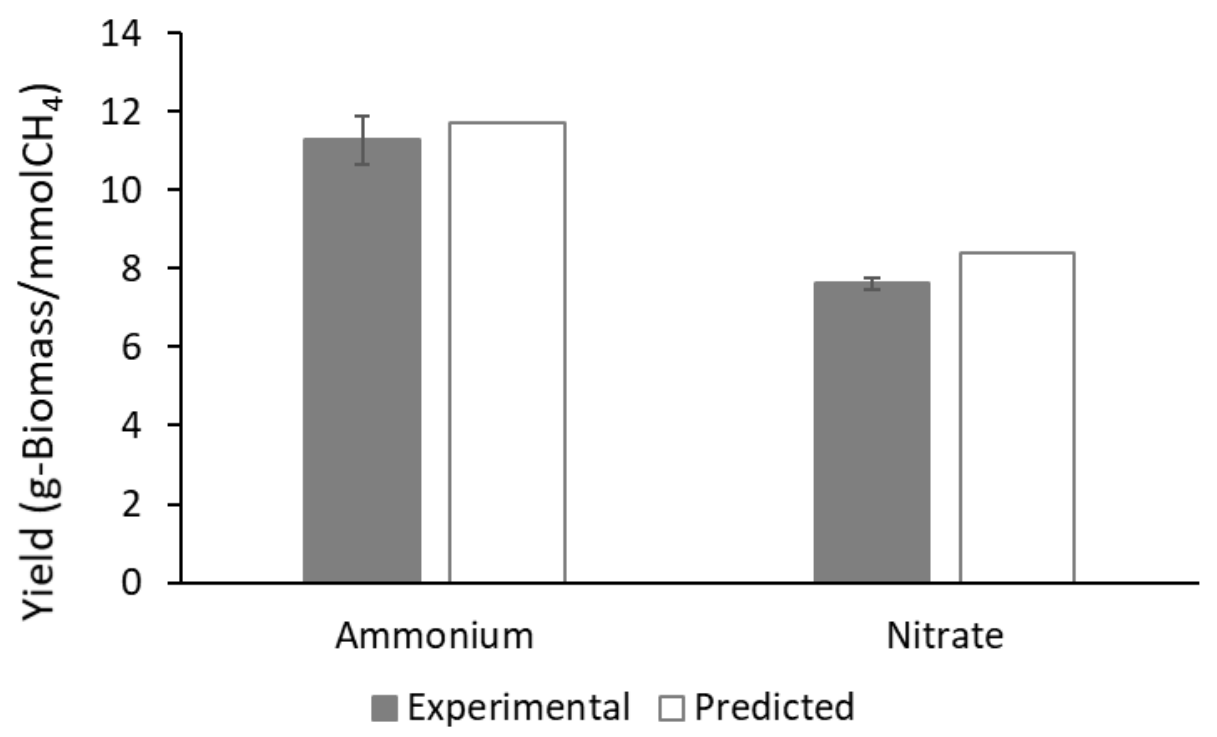

Figure 4. Predicted and experimental biomass yields using nitrate and ammonium as nitrogen sources in M. hirsuta. Ammonium resulted in higher yields on methane but at the expense of lower growth rates due to competitive inhibition of the pMMO enzyme. Grey bars correspond to the observed experimental values, with error bars equal to the standard deviations (for 3 biological replicates). White bars correspond to predictions using the GSMM of Methylocystis hirsuta.

Yields on methane using ammonium and nitrate as nitrogen sources were also measured for Methylocystis sp. SC1 (see supplementary figure S1), the obtained yields were practically identical to the theoretical ones.

\subsubsection{Production of PHB under nitrogen starvation conditions.}

Methylocystis hirsuta can accumulate PHB over $40 \%$ of its total biomass dry weight under conditions of complete nitrogen depletion (López et. al., 2018). By monitoring methane consumption and PHB accumulation over time, it was found that $M$. hirsuta consumed methane at a specific rate of $46 \pm 1 \mathrm{mmol} \mathrm{g}_{-} \mathrm{DW}^{-1}$ day $^{-1}$ under nitrogen deprivation. The experimental yield of PHB on methane was found to be $0.088 \pm 0.002 \mathrm{molPHB} / \mathrm{molCH}_{4}$. Using the experimental specific methane consumption and ATP maintenance consumption rates as constraints, the optimization of PHB resulted in an estimated value of $0.11 \mathrm{molPHB} / \mathrm{molCH}_{4}$ with an oxygen-methane consumption ratio of $1.5 \mathrm{molO}_{2} / \mathrm{molCH}_{4}$ (the same as in the case of biomass production), compared to an experimental value of $1.52 \pm 0.012 \mathrm{molO}_{2} / \mathrm{molCH}_{4}$. Figure 
5 shows the predictions and experimental results for PHB yield on methane and oxygenmethane consumption ratio.

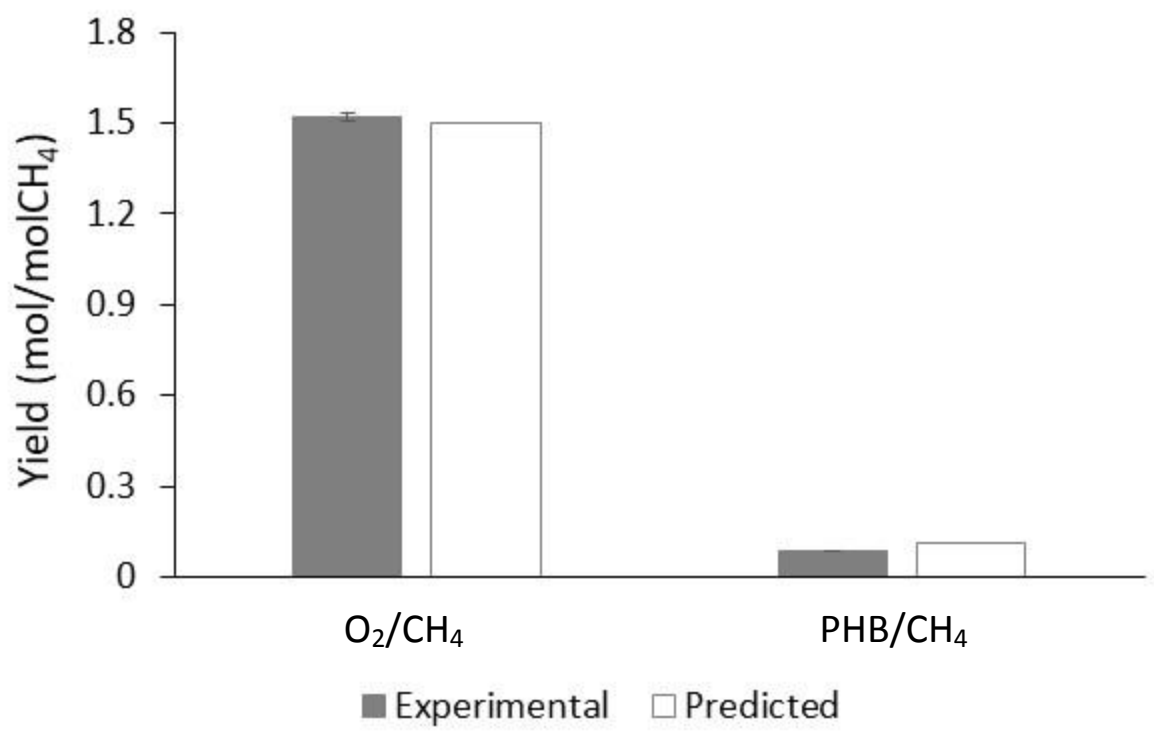

Figure 5. Theoretical versus experimental PHB yield on methane and oxygen-methane consumption molar ratios. Grey bars correspond to the observed experimental values, with error bars equal to the standard deviations (for 3 biological replicates). White bars correspond to predictions using the GSMM of Methylocystis hirsuta.

\subsection{Metabolic capability of M. hirsuta to supply building blocks for biosynthesis.}

A large variety of industrially relevant products can be synthesized by $M$. hirsuta from a small number of metabolic precursors (Jouhten et. al., 2016), some key precursors and final products are summarized in Table 3.

Table 4. Metabolic precursors and derived industrial products

\begin{tabular}{l|l}
\hline \multicolumn{1}{c}{ Metabolic precursor } & \multicolumn{1}{c}{ Industrial products } \\
\hline 3-Dehyidroshikimate & Vaniline \\
\hline L-Tyrosine & $\begin{array}{l}\text { Pinocembrin, eriodictyol, naringenin, resveratrol, } \\
\text { homoeriodicytol, chrisyn, luteolin, apigentin. }\end{array}$ \\
\hline Pyruvate & Lactate \\
\hline
\end{tabular}




\begin{tabular}{l|l}
\hline Oxaloacetate & Nicotinamine, 4-methylthiobutyl-desulfoglycosinolate. \\
\hline Glyceraldehyde-3P & Propane-1,2-diol, propane-1,3-diol, \\
\hline Acetyl-CoA & 6-methylsalicylate \\
\hline Succinate & Succinate \\
\hline Aceto-Acetyl-CoA & $\begin{array}{l}\text { PHB, butanol, geraniol, beta-carotene, valencene, 8-epi- } \\
\text { cedrol, cubebol, patchoulol, amorpha-4,11-diene }\end{array}$ \\
\hline
\end{tabular}

For each of these final products, there are metabolic pathways that could be expressed in the host organism and which would drain the corresponding precursor in the central carbon metabolism (their production would also be associated with the consumption of ATP and redox cofactors, therefore the estimations presented here are just meant to be indicative). Thus, biomass production (or PHB production in the case of aceto-acetyl-CoA) was first optimized using the reconstructed GSMMs. A parsimonious (minimizing the sum of metabolic fluxes) optimal solution was obtained using COBRApy and only the reactions active in this solution were allowed to carry flux in the subsequent optimizations. This was done in order to identify the metabolic sub-network that was active under optimal growth conditions (in the presence of nitrogen) and under optimal PHB synthesis (in the absence of nitrogen). Therefore, the results depicted in Figure 6 were meant to represent the maximal amounts of each precursor that the introduction of a new pathway could drain from the active metabolic network of the cell (without extra metabolic enzymes having to be overexpressed). All the three metabolic networks resulted in identical results, suggesting that the architecture of the central carbon metabolism is very similar among the three species of Methylocystis. 
A
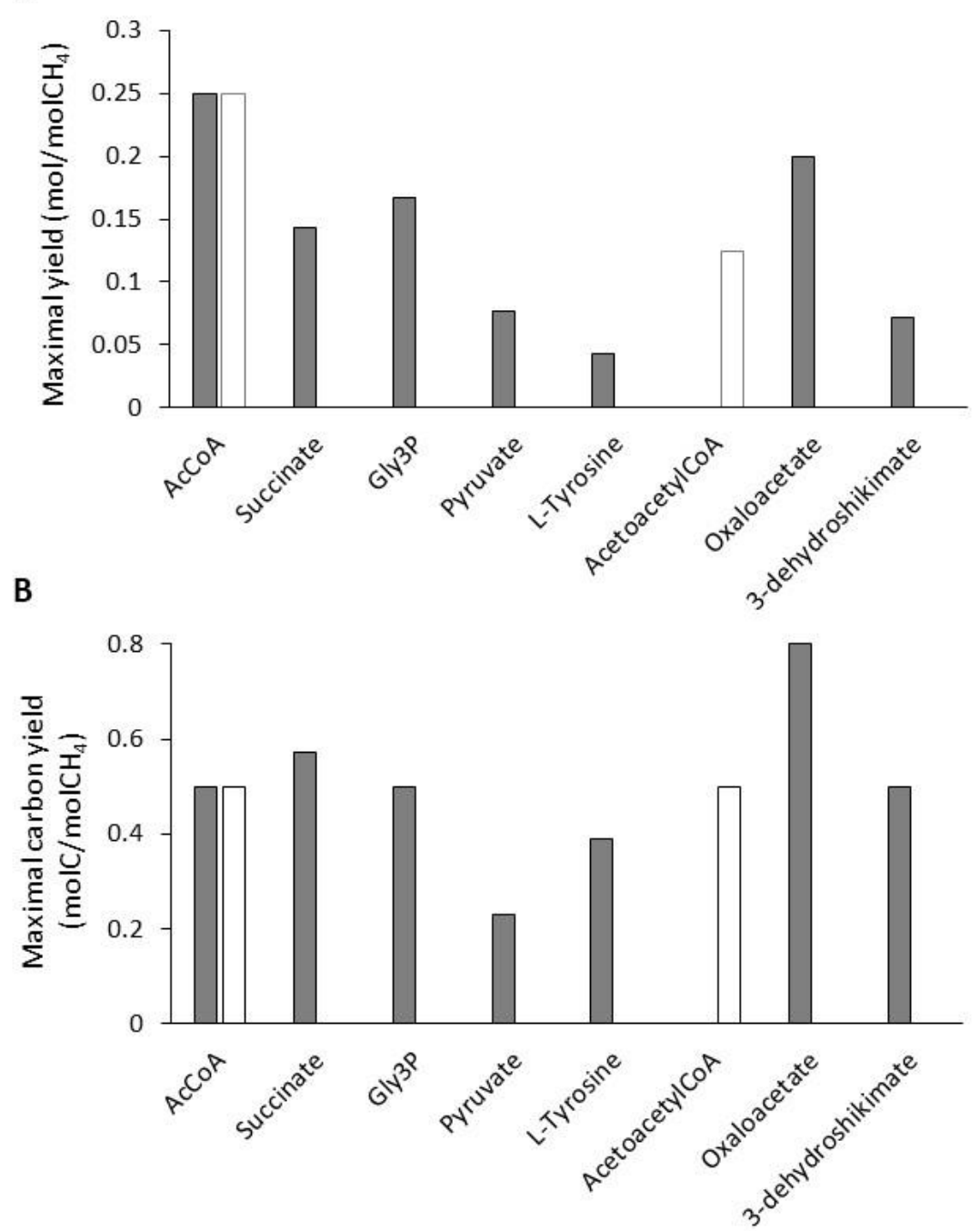

Figure 6. Maximal yields of metabolic precursors in the presence (grey bars) and absence of nitrogen (white bars). Molar yields on methane (A) and carbon yields on methane (B). Each yield has been calculated by fixing the methane uptake rate to its experimental value for Methylocystis hirsuta, setting to zero the rates of the reactions non-used for growth optimization (grey bars), or PHB optimization (white bars), and maximizing the production rate of each metabolic precursors. In the cases of Acetyl-CoA and Acetoacetyl-CoA, the cofactor is previously removed from acetate and acetoacetate respectively (so that there is no net loss of CoA).

As it could be expected, the precursor acetoacetyl-CoA is produced under nitrogen limitation conditions. The fact that Methylocystis strains are able to deviate high metabolic fluxes towards PHB (and therefore towards its precursor acetoacetyl-CoA) under nitrogen starvation, makes these operation conditions particularly suitable for the production of any of the derivatives of acetoacetyl-CoA (table 3). In terms of total carbon yields, oxaloacetate was the precursor most efficiently produced, which makes these species potential cell factories for the production of 
chemicals such as nicotinamine or 4-methylthiobutyl-desulfoglycosinolate. Succinate, which besides being a metabolite of the central carbon metabolism is also a very important building block for the chemical industry, can also be produced at high carbon yields. A simple overexpression of a suitable membrane transporter or the knockout of succinate consuming reactions, could make these strains suitable succinate producers.

\subsection{Utilization of C2 compounds as carbon source.}

The strain Methylocystis sp. SB2 is known to be able to grow on C2 carbon sources such as acetate or ethanol (Im et. al., 2011). Acetyl-CoA can support the production of NADH through the TCA cycle, however it is not able to sustain the anaplerotic function of the TCA cycle as supplier of precursors for biosynthesis. Therefore, in order to use C2 carbon sources, the cell must transform Acetyl-CoA into C3 compounds such as glycerate, which can be transformed into precursors for biosynthesis (via the lower glycolysis). Methylocystis sp. SB2 relies on a malonyl-CoA lyase to transform $\beta$-methylmalonyl-CoA into glyoxylate and propionyl-CoA. Glyoxylate is then transformed into glycerate via the so-called glyoxylate assimilation cycle. This mechanism has been confirmed in Methylocystis sp. SB2 by RNA-seq results, which showed the overexpression of all the metabolic genes involved in the glyoxylate assimilation cycle during growth on C2 substrates (Vorobev et. al., 2014). In this context, the models of Methylocystis hirsuta and Methylocsytis sp. SC2 included all the necessary reactions for glyoxylate assimilation, in particular the enzyme malonyl-CoA lyase. Therefore, it is reasonable to expect that all the three strains exhibit the potential to grow on $\mathrm{C} 2$ substrates.

The ability of Methylocystis hirsuta to grow on acetate was assessed with very low specific growth rates $\left(0.08 \pm 0.006\right.$ day $\left.^{-1}\right)$ and a biomass yield on acetate of $14 \pm 2 \mathrm{~g}-\mathrm{DW} \mathrm{mol}^{-1}$. This was very similar to the yield of $12 \pm 1 \mathrm{~g}-\mathrm{DW} \mathrm{mol}^{-1}$ reported for Methylocystis sp. SB2 (Im et. al., 2011), but lower than the maximal theoretical yield of $17 \mathrm{~g}-\mathrm{DW} \mathrm{mol}^{-1}$ predicted by the GSMM. It has been hypothesized that acetate has an uncoupling role in the respiratory chain, leading to lower respiration efficiencies (Im et. al., 2011). Despite the poor growth rates and biomass 
yields observed, the GSMM here developed suggests that Methylocystis hirsuta is indeed able to use C2 carbon compounds as carbon and energy sources.

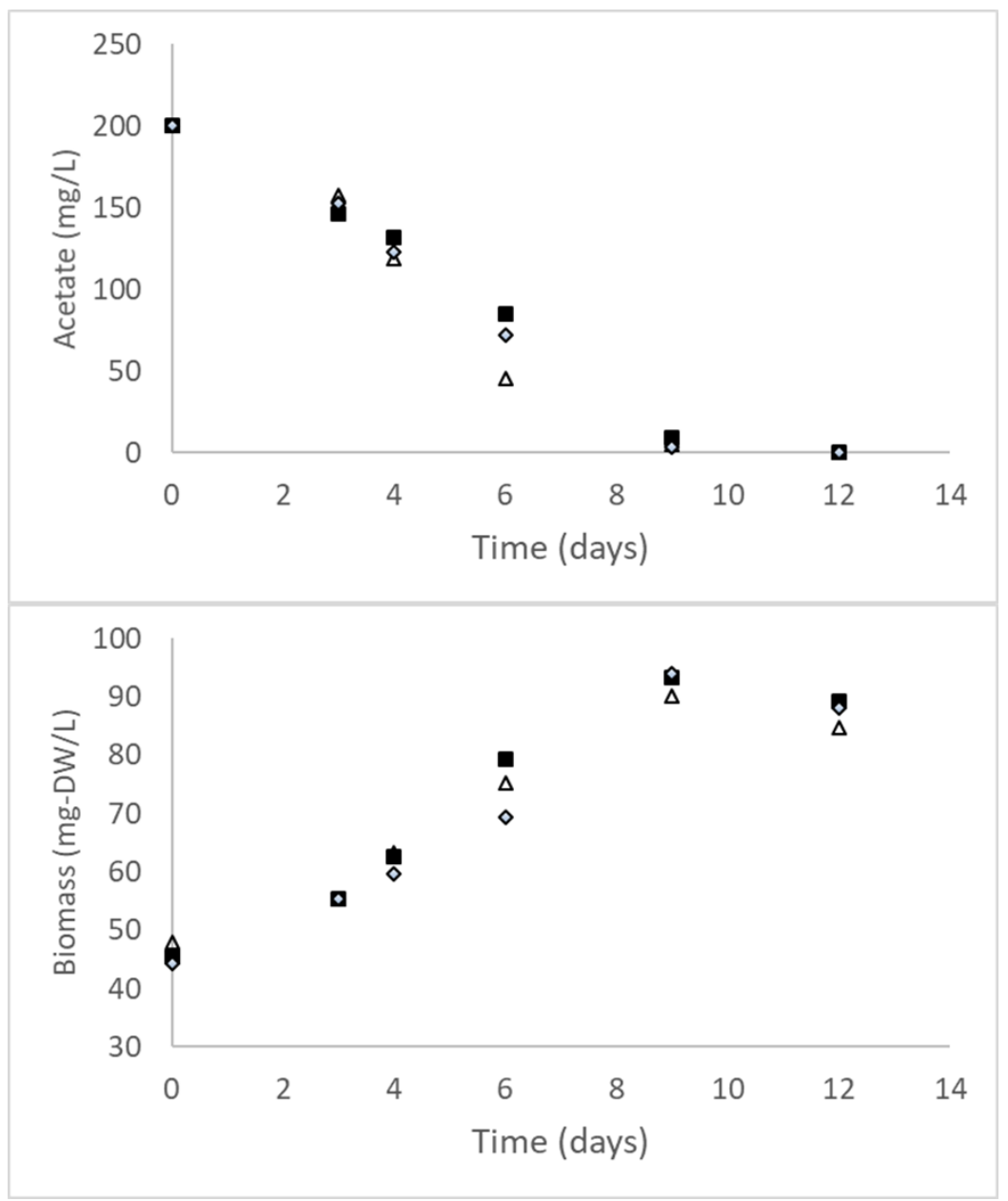

Figure 7. Growth and acetate consumption curves of Methylocystis hirsuta. Three biological replicates are shown. The observed biomass yield on acetate $14 \pm 2 \mathrm{~g}-\mathrm{DW} \mathrm{mol}^{-1}$ was similar to the value reported for Methylocistis sp SB2.

Growth on acetate was also tested for Methylocystis sp. SC2 (see supplementary figure S2). After 400 hours the OD of the culture increased from 0.074 to 0.081 , even if the difference was statistically significant ( 3 replicates), it cannot be concluded from the results that this strain is able to grow on $\mathrm{C} 2$ substrates.

\section{Discussion}

The reconstruction of GSMMs for M. hirsuta, M. sp. SC2 and M. sp. SB2 revealed a very similar metabolism, with over $90 \%$ shared metabolic reactions and metabolites. The experimental biomass yields and oxygen-methane consumption ratios observed in Methylocystis hirsuta matched the predictions obtained with the GSMM under the assumption of maximal 
biomass production and the "redox branch" mechanism for methane oxidation. The elucidation of the methane oxidation mechanism has been one of the main topics of controversy in the field of methanotrophy. Previous results with Type I methanotrophs pointed out to the "direct coupling" as the most likely mechanism (de la Torre et. al., 2015; Akberdin et. al., 2018). However, our results suggested that the nature of the redox co-factor involved in methane oxidation could be another of the key differences between Type I and II methanotrophs. The results for Methylocystis hirsuta regarding the methane oxidation mechanisms can very likely be extrapolated to the other two Methylocystis strains, as the pMMO enzymes coded in their genomes appeared to be very similar or identical (Bordel, et. al., 2018).

The experimental biomass yields of Methylocystis hirsuta were close to its maximal theoretical yields (both in the case of nitrate and ammonium used as carbon sources). This assumption of optimal yields, even if it is broadly used as a modeling approach for microorganisms, is often not accurate under substrate excess conditions (Bordel, 2013). The growth rate of methanotrophs of the genus Methylocystis seems to be limited by the rate of methane oxidation. This was confirmed in the case of Methylocystis sp. SC2 by the fact that knocking out one of the two identical copies of a pMMO contained in the genome resulted in a decrease of its growth rate (Baani and Liesack, 2018). Organisms limited by substrate availability (or utilization) tend to have evolved to use substrates as efficiently as possible, leading to yields close to their theoretical maximum. Interestingly, the observed PHB yields under nitrogen deprivation conditions were also close to the theoretical maximum predicted by the model.

It was also confirmed that $M$. hirsuta, as well as its relative $M$. sp. SB2, is able to grow on $\mathrm{C} 2$ carbon sources (acetate in this case) by using the glyoxylate assimilation pathway to transform acetyl-CoA into glycerate. However, the observed growth rate on acetate was dramatically lower than the growth rate on methane (17 times lower), which suggests that this pathway, even if it exists, is rather inefficient. This inefficiency at transforming acetyl-CoA into building blocks for biosynthesis reveals that the methane assimilation pathway in Type II 
methanotrophs, the serine cycle, is more complex than its usual textbook descriptions. Indeed, the serine cycle yields acetyl-CoA as a product, which itself can be used to fuel the TCA cycle and obtain energy but not as a biomass building block (or at least not efficiently, as we have previously discussed). The flux distributions predicted by the reconstructed models revealed that part of the serine produced in the serine cycle is transformed into L-cysteine by a cystathionine $\beta$-synthase and this L-cysteine is the substrate for the biosynthesis of all the necessary biomass building blocks. In order to keep the concentrations of the intermediates of the serine cycle under steady state, glycine is supplied into the cycle by a glycine synthase that transforms methylene-tetrahydrofolate into glycine, playing an anaplerotic role in the serine cycle similar to the role played by pyruvate carboxylase in the TCA cycle (Figure 7). This high flux through L-cysteine should be considered for future metabolic engineering strategies using Methylocystis as cell factories.

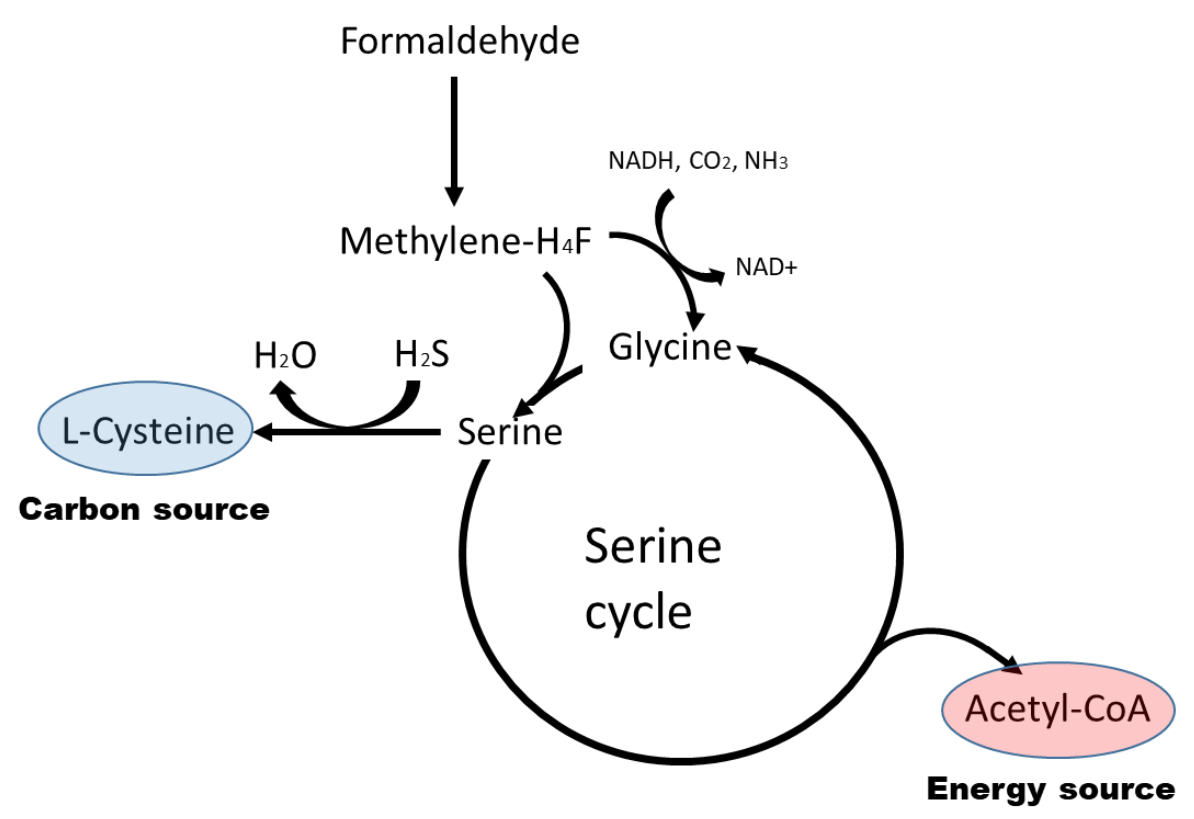

Figure 8. Illustration of the serine cycle producing acetyl-CoA for the TCA cycle and L-cysteine for biosynthesis of biomass building blocks. For the serine cycle to operate in steady state, the serine removed for the synthesis of Lcysteine has to be compensated by supplying glycine into the cycle by glycine synthase. In absence of this reaction, the serine cycle could operate supplying Acetyl-CoA (fueling the TCA cycle and respiration) but there would not be net production of precursors for biosynthesis. 
Finally, Flux Balance Analysis simulations were performed in order to assess the potential of the Methylocystis strains here analyzed to supply precursors for the biosynthesis of chemicals of industrial and commercial interest. These simulations revealed that the Methylocystis strains have a considerable potential for the production of chemicals such as succinate, acetoacetylCoA derivatives (under nitrogen starvation conditions) and oxaloacetate derivatives.

\section{Acknowledgements}

This work was performed with the support of the Marie Curie grant H2020-MSCA-IF-2016 CH4BioVal (GA no 750126). The financial support of the Regional Government of Castilla y León and the FEDER program and the Ministry of Science, Innovation and Universities are also gratefully acknowledged (CLU 2017-09, CTM2015-70442-R and VA281P18)

\section{References:}

Abbasi, T., Tauseef, S.M, Abbasi, S.A., 2012. Anaerobic digestion for global warming control and energy generation, an overview. Renew. Sustain. Energy. Rev. 16, 3228-3242.

Akberdin, I.R., Thompson, M., Hamilton, R., Desai, N., Alexander, D., Henard, C.A., Guarnieri, M.T., Kalyuzhnaya, M.G., 2018. Methane utilization in Methylomicrobium alcaliphilum $20 Z^{\mathrm{R}}$ : a systems approach. Sci. Rep. 8, 2512.

Baani, M., Liesack, W. 2008. Two isozymes of particulate methane monooxygenase with different methane oxidation kinetics are found in Methylocystis sp. strain SC2. Proc. Natl. Acad. Sci. USA. 105, 10203-10208.

Becker, S.A., Price, N.D., Palsson, B.O., 2006. Metabolite coupling in genome-scale metabolic networks. BMC Bioinformatics. 7, 111.

Bordel, S., 2013. Experimental evidence suggests the existence of evolutionary conserved global operation principles governing global metabolism. Sci. Rep. 3, 3017. 
Bordel, S., Rodríguez, E., Muñoz, R., 2018. Genome sequence of Methylocystis hirsuta CSC1, a polyhydroxyalkanoate producing methanotroph. MicrobiologyOpen. 7, e771.

Campbell, M.A., Nyerges, G., Kozlowski, J.A., Poret-Peterson, A.T., Stein L.Y., Klotz, M.G., 2011. Model of the molecular basis for hydroxylamine oxidation and nitrous oxide production in methanotrophic bacteria. FEMS Microbiol Lett. 322, 82-89.

Chelliah, V., Juty, N., Ajmera, I., Ali, R., Dumousseau, M., Glont, M., Hucka, M., Jalowicki, G., Keating, S., Knight-Schrijver, V., Lloret-Villas, A., Natarajan, K.N., Pettit, J.B., Schubert, M., Wimalaratne, S.M., Zhao, Y., Hermjakob, H., Le Novere, N., Laibe, C., 2015. BioModels: ten-year anniversary. Nucl. Acids Res. 43, D542-D548.

Comer A.D, Long M.R, Reed J.L, Brian F.P., 2017. Flux balance analysis indicates that methane is the lowest cost feedstock for microbial cell factories. Metab. Eng. Commun. 5, 2633.

Dam, B., Dam, S., Kube, M., Reinhardt, R., Liesack, W., 2012. Complete genome sequence of Methylocystis $s p$. Strain SC2, an aerobic methanotroph with high affinity methane oxidation potential. J. Bacteriol. 194, 6008-6009.

Dam, B., Dam, S., Blom, J., Liesack, W., 2013. Genome analysis coupled with physiological studies reveals a diverse nitrogen metabolism in Methylocystis sp. strain SC2. PLoS ONE 8, e74767.

Degli Esposti, M., 1998. Inhibitors of NADH-ubiquinone reductase: an overview. Biochim. Biophys. Acta. 1364, 222-235.

Ebrahim, A., Lerman, J.A., Palsson, B.O., Hyduke, D.R., 2013. COBRApy: COnstraints-Based Reconstruction and Analysis for Python. BMC Syst. Biol. 7, 74.

García-Pérez, T., López, J.C., Passos, F., Lebrero, R., Revah, S., Muñoz, R. 2018. Simultaneous methane abatement and PHB production by Methylocystis hirsuta in a novel gas-recycling bubble column bioreactor. Chem. Eng. J. 334, 691-697. 
Henard, C.A., Smith, H., Dowe, N., Kalyuzhnaya, M.G., Pienkos, P.T., Guarnieri, M.T., 2016. Bioconversion of methane to lactate by an obligate methanotrophic bacterium. Sci. Rep. 6 , 21585.

Im J., Lee, S.W., Yoon, S., DiSprito, A.A., Semrau, J.D., 2011. Characterization of a novel facultative Methylocystis species capable of growth on methane, acetate and ethanol. Environ. Microbiol. Rep. 3, 174-181.

Jouhten, P., Boruta, T., Andrejev, S., Pereira, F., Rocha, I., Patil, K.R., 2016. Yeast metabolic chassis designs for diverse biotechnological products. Sci. Rep. 6, 29694.

Kalyuzhnaya, M.G., Puri, A.W., Lidstrom, M.E., 2015. Metabolic engineering in methanotrophic bacteria. Metab. Eng. 29, 142-152.

López, J.C., Quijano, G., Pérez, R., Muñoz, R., 2014. Assessing the influence of CH4 concentration during culture enrichment on the biodegradation kinetics and population structure. J. Environ. Manage. 146, 116-123.

Kalyuzhnaya, M.G., Khmelenina, V., Eshinimaev, B., Sorokin, D., Fuse, H., Lindsgrom, M., Trotsenko, Y., 2008. Classification of halo(alkali)philic and halo(alkali)tolerant methanotrophs provisionally assigned to the genera Methylomicrobium and Methylobacter and emended description of the genus Methylomicrobium. Int. J. Syst. Evol. Microbiol. 58, 591-596.

López, J.C., Arnáiz, E., Merchán, L., Lebrero, R., Muñoz, R., 2018. Biogas-based polyhydroxyalkanoates produced by Methylocystis hirsuta: A step further in anaerobic digestión biorefineries. Chem. Eng. J. 333, 529-536.

Nguyen, A.D., Hwang, I.Y., Lee, O.K., Kalyuzhnaya, M.G., Mariyana, R., Hadiyati, S., Kim, M.S., Lee, E.Y., 2018. Systematic metabolic engineering of Methylomicrobium alcaliphilum $20 \mathrm{Z}$ for 2,3-butanediol production from methane. Metab. Eng. 47, 323-333.

Overbeek, R., Begley, T., Butler, R.M., Choudhuri, J.V., Chuang, H.Y., Cohoon, M., de CrécyLagard, V., Diaz, N., Disz, T., Edwards, R., Fonstein, M., Frank, E.D., Gerdes, S., Glass, E.M., Goesmann, A., Hanson, A., Iwata-Reuyl, D., Jensen, R., Jamshidi, N., Krause, L., Kubal, M., 
Larsen, N., Linke, B., McHardy, A.C., Meyer, F., Neuweger, H., Olsen, G., Olson, R., Osterman, A., Pornov, V., Pusch, G.D., Rodionov, D.A., Rückert, C., Steiner, J., Stevens, R., Thiele, I., Vassieva, O., Ye, Y., Zagnitko, O., Vonstein, V., 2005. The subsystems approach to genome annotation and its use in the project to annotate 1000 genomes. Nucleic Acids Res. 33, 5691- 5702

Overbeek, R., Olson, R., Pusch, G.D., Olsen, G.J., Davis, J.J., Disz, T., Edwards, R.A., Gerdes, S., Parrello, B., Shukla, M., Vonstein, V., Wattam, A.R., Xia, F., Stevens, R., 2014. The SEED and the rapid annotation of microbial genomes using subsystems technology (RAST). Nucleic Acids Res, 42, D206-D214

Patil. K.R., Rocha, I., Förster, J., Nielsen, J., 2005. Evolutionary programming as a platform for in silico metabolic engineering. BMC Bioinform. 6, 308

Pieja, A.J., Morse, M.C., Cal, A.J., 2017. Methane to bioproducts: the future of the bioeconomy? Curr. Opin. Chem. Biol. 41, 123-131.

Puri, A.W., Owen, S., Chu, F., Chavkin, T., Beck, D.A.C., Kalyuzhnaya, M.G., Lindstrom, E., 2015. Genetic tools for the industrially promising methanotroph Methylomicrobium buriatense. Appl. Environ. Microbiol. 81, 1775-1781.

Strong, P.J., Kalyuzhnaya, M., Silverman, J., Clarke, W.P., 2016. A methanotroph-based biorefinery: Potential scenarios for generating multiple products from a single fermentation. Bioresour. Technolol. 215, 314-323.

de la Torre, A., Metivier, A., Chu, F., Laurens, L.M.L., Beck, D.A.C., Pienkos, P.T., Lindstrom, M.E., Kaluzhnaya, M.G., 2015. Genome-scale metabolic reconstruction and theoretical investigation of methane conversión in Methylomicrobium buryatense strain 5G(B1). Microb. Cell. Fact. 14, 188.

Vorobev, A., Jagadevan, S., Jain, S., Anantharaman, K., Dick, G.J., Vuilleumier, S., Semrau, J.D., 2014. Genomic and transcriptomic analyses of the facultative methanotroph Methylocystis $s p$. strain SB2 grown on methane or ethanol. Appl. Environ. Microbiol. 80, 3044-3052. 
Whittenbury, R., Phillips, K.C., Wilkinson., J.F., 1970. Enrichment, isolation and some properties of methane-utilizing bacteria. J. Gen. Microbiol. 61, 205-218.

Zuniga, C., Morales, M., Le Borgne, S., Revah, S., 2011. Production of poly- $\beta$-hydroxybutyrate by methylobacterium organophilum isolated form a methanotrophic consortium in a two phasepartition bioreactor. J. Hazard. Mater. 190, 876-882. 